\title{
Araujo, Ana Lucia. - Public Memory of Slavery
}

\section{Emmanuelle Kadya Tall}

\section{(2) OpenEdition}

Journals

Édition électronique

URL : http://journals.openedition.org/etudesafricaines/14834

DOI : 10.4000/etudesafricaines. 14834

ISSN : $1777-5353$

\section{Éditeur}

Éditions de l'EHESS

\section{Édition imprimée}

Date de publication : 22 novembre 2013

Pagination : 935-938

ISBN : 978-2-7132-2389-1

ISSN : 0008-0055

\section{Référence électronique}

Emmanuelle Kadya Tall, «Araujo, Ana Lucia. - Public Memory of Slavery », Cahiers d'études africaines [En ligne], 212 | 2013, mis en ligne le 06 décembre 2013, consulté le 24 septembre 2020. URL : http:// journals.openedition.org/etudesafricaines/14834; DOI : https://doi.org/10.4000/etudesafricaines. 14834

Ce document a été généré automatiquement le 24 septembre 2020.

(c) Cahiers d'Études africaines 


\title{
Araujo, Ana Lucia. - Public Memory of Slavery
}

\author{
Emmanuelle Kadya Tall
}

\section{RÉFÉRENCE}

ARAujo, Ana Lucia. - Public Memory of Slavery. Victims and Perpetrators in the South Atlantic. Amherst-New York, Cambia Press, 2010, 478 p., bibl.

1 La lecture de l'ouvrage dense et très didactique d'Ana Lucia Araujo (sept chapitres suivis chacun d'un résumé) nous rappelle notre premier séjour au Bénin, au milieu des années 1980, lorsque le régime marxiste-léniniste de Mathieu Kérékou arrivait à son terme. Un précédent terrain dans une société hiérarchisée en groupes statutaires, dans laquelle le groupe des esclaves continuait à porter les stigmates de la condition servile, nous faisait observer avec étonnement le dédain avec lequel les sociétés du Bénin méridional abordaient la question de l'esclavage. À l'époque, on nous renvoyait inéluctablement à la stratification sociale des Aguda, ces anciens esclaves brésiliens retournés en Afrique ayant compagnonné avec les négriers portugais dont ils partageaient, en dépit de leur statut diamétralement opposé, une culture occidentale. Quant à la traite atlantique et l'agency des pourvoyeurs d'esclaves aux négriers européens, le silence était de mise et si, lors de la visite du musée d'Abomey, ancienne capitale du royaume du même nom, les guides se délectaient à décrire, avec force détails, la coutume d'enterrer le roi avec son cortège de femmes suivi de ses meilleurs esclaves, il n'y avait ni remords, ni contrition, mais au contraire une certaine fierté à exhiber ce qui symbolisait la puissance et la richesse de l'ancien royaume.

2 La question de l'esclavage faisait d'autant moins question que, contrairement à ce que nous avions pu constater au Nord-Sénégal, le statut de descendant d'esclave ne portait pas à des jugements. L'esclavage issu de la traite atlantique semblait un épiphénomène sans conséquence pour le Bénin contemporain et l'animosité que certains pouvaient avoir vis-à-vis des Aguda, tenait moins à leur qualité d'ancien négrier ou d'ancien 
esclave parvenu, qu'à la complicité de ces Brésiliens qui avaient su pactiser avec le pouvoir colonial pour obtenir des avantages substantiels. Interprètes et commis de l'administration coloniale, ils avaient su tirer parti de leur connaissance du monde occidental en constituant une partie de cette élite intellectuelle qui fera du Dahomey postcolonial, le quartier latin de l'Afrique.

Revenant à l'ouvrage, dès l'introduction, Ana Lucia Araujo pose les fondements de sa problématique, à savoir le rapport complexe entretenu entre mémoire et histoire, et son évocation des historiens de la mémoire (Ricœur, Nora) et de la mise en patrimoine qui caractérise notre monde contemporain (Hartog) en témoigne bien. Le premier chapitre est consacré à une revue critique de l'historiographie de l'esclavage qui, d'une part et jusqu'aux années 1960, a appréhendé l'esclavage en Afrique et l'esclavage aux Amériques comme deux objets distincts et qui, d'autre part, dans ses recherches s'est peu intéressée aux archives non occidentales ou à l'expérience de l'esclavage qui ne sera appréhendée comme objet d'étude qu'à partir des années 1980. Dans un deuxième chapitre, consacré aux mémoires plurielles de l'esclavage et de la traite atlantique, l'auteure passe en revue les débats académiques européens, africains et américains qui tournent principalement autour de la démographie de l'esclavage, contrairement aux débats sur la mémoire qui se tiennent dans l'arène publique et sont toujours fortement médiatisés. Le talent du gardien de la Maison des Esclaves à Gorée est mis en avant dans la construction du mythe de Gorée, aujourd'hui mis à mal par les travaux archéologiques récents, et l'auteure remarque avec justesse que l'histoire et la mémoire sont souvent contradictoires.

4 Le troisième chapitre examine la spécificité de l'esclavage au Brésil qui, au fur et à mesure de l'évolution de la traite atlantique, se traduit par la prise de distance des Portugais du Brésil vis-à-vis de la métropole, en traitant directement avec les différents royaumes de la Baie du Bénin et en particulier avec le royaume du Dahomey. Ces échanges Sud-Sud ont formé de véritables couloirs de continuité entre les sociétés dahoméennes et bahianaises. Cependant, s'il est vrai que le XIX ${ }^{e}$ siècle avec le retour de certains esclaves affranchis sur les côtes africaines a donné naissance à un flux de personnes, d'artefacts et d'idées entre les deux côtes atlantique, il nous semble que la réactivation mémorielle dont elle est l'objet aujourd'hui est, comme le note l'auteure, toujours impulsée de l'extérieur, soit par une institution internationale, soit par une personnalité : le rôle de Pierre Verger de ce point de vue est assez édifiant en dépit de l'insuccès relatif de ses entreprises : la maison du Brésil à Ouidah survit difficilement et la maison du Bénin à Bahia est devenue un simple restaurant.

Depuis le renouveau démocratique en 1991, les processus de patrimonialisation de la mémoire de l'esclavage et la question de l'esclavage, s'ils font débat dans la sphère publique, continuent de renvoyer aux Aguda, comme le note justement l'auteure en s'appuyant sur les réflexions de l'historien béninois Elisée Soumonni. Ce dernier remarque que, loin d'être dans une période pacifiée comme le pensait le photographe devenu anthropologue, Milton Guran, les Aguda sont au contraire en train de se déchirer entre victimes et acteurs de la traite atlantique. Il y aurait maintenant les vilains acteurs de la Traite et ses victimes. Le cas de Zoki Kata, originaire de Hoko dans le pays Mahi et esclave déporté à Bahia puis Retornado installé à Agoué, témoigne du fait que la frontière entre victime et acteur de la traite atlantique est des plus problématiques : en effet, en faisant le chemin du retour, Zoki alias Joaquim de Almeida 
se livre à la traite des esclaves avant de se reconvertir dans l'économie de plantation, utilisant à son tour une main-d'œuvre esclave.

6 Le chapitre 4 intitulé "Mémoires contestées de la traite esclavagiste au Bénin méridional » rend compte des commémorations internationales de la Route des Esclaves et du Festival Ouidah 92 avec les paradoxes qu'a pu suscité la célébration conjointe des étapes plus ou moins reconstruites, voire inventées, de la route menant les esclaves vers la plage d'embarquement pour les territoires du Nouveau monde, et de celle de l'ancien royaume esclavagiste aboméen.

7 Le chapitre 5 nous conduit dans l'Afrique du Brésil. Constatant qu'au Bénin, le réveil d'une mémoire de l'esclavage est le fait des organisations internationales et du pouvoir politique après le retour à la démocratisation, l'auteure souligne combien au Brésil, ce sont les luttes sociales et religieuses qui, à partir des années 1990, impulsent la mise en place de politiques sociales de discrimination positive à l'égard des segments afrobrésiliens de la population. Avant cela, notamment à partir des années 1980, c'est à travers la musique, le Carnaval et la religion que la population afro-brésilienne exprime son identité africaine. L'auteure semble regretter que, malgré la mise en place de politiques de discrimination positive, le Brésil en tant que nation édifie si peu de monuments à la gloire des résistants à l'esclavage, tout en soulignant combien l'image de l'esclavage a évolué chez ses descendants de la victimisation à la résistance. Si l'on peut en effet regretter l'absence de ces nouvelles figures de la résistance dans la mise en monuments de héros afro-brésiliens, on s'étonnera toutefois de la vision romantique voire irénique qu'elle donne de l'ancien Dahomey dans la mesure où, dans ce pays comme au Brésil, le déni de l'esclavage y est tout aussi tenace. En effet, la construction de monuments mémoriaux à Ouidah et sur sa plage était moins destinée à la mémoire des populations locales qu'à celle de la diaspora américaine. Et si aujourd'hui les populations locales se les réapproprient dans leurs négociations sociales, c'est en vertu de leurs propriétés monnayables sur le vaste marché global de la mémoire et du patrimoine.

8 Le chapitre 6 est consacré à l'héritage de Francisco Felix de Souza, le marchand négrier allié au roi Guezo dans le développement du comptoir de Ouidah et le passage de l'économie de la Traite à l'économie de plantation. Soulignant combien il demeure la figure phare $\mathrm{du}$ Brésilien négrier mais philanthrope, en dépit de la nouvelle historiographie qui montre combien il était loin d'être le marchand le plus puissant et le plus riche de l'ancienne côte des Esclaves, A. L. Araujo suggère que son succès aujourd'hui tient en grande partie à la capacité de ses descendants à réactiver des liens transnationaux. L'auteure, elle-même Brésilienne du Brésil, relie la brésilianité des descendants de cet illustre fondateur de la communauté aguda à Ouidah à l'imaginaire des trois races au Brésil. Cependant, malgré la réactualisation des liens entre le Brésil et l'ancien Dahomey au milieu du $\mathrm{XX}^{\mathrm{e}}$ siècle qui peut avoir véhiculé cette image de cordialité raciale au sein de la communauté aguda ou de ses élites, nous doutons qu'elle soit localement généralisable. Et ce d'autant moins que, jusqu'à aujourd'hui, le trait marquant de la brésilianité aguda au Bénin s'exprime dans la question consacrée que l'on pose au prétendant étranger à la communauté aguda qui viendrait demander la main d'une Aguda: Seras-tu capable de lui donner à manger du beurre? Toutefois l'auteure a raison de souligner l'importance des projets internationaux et des processus de démocratisation et de décentralisation dans la réémergence du lignage de Souza et des Aguda en général dans la sphère sociopolitique contemporaine. 
9 Le dernier chapitre intitulé « Oublier et se souvenir du passé esclavagiste » est consacré à l'analyse des mémoires publiques de l'esclavage et du Brésil chez deux personnalités aguda. L'auteure, réaffirmant qu'en Afrique, contrairement à l'Europe ou les Amériques, l'esclavage constitue un stigma social, pense trouver là l'explication du fait que les descendants des anciens esclaves retournés au Bénin, loin de se poser collectivement en victimes, agissent individuellement pour promouvoir leur ancestralité brésilienne. On pourrait adopter cette explication si les personnalités dont il s'agit n'étaient pas des figures éminentes de la vie socio-économique du Bénin contemporain. Entre l'homme d'affaires aux activités multiples et le frère de la première dame du renouveau démocratique au Bénin, l'affirmation identitaire d'ancien esclave « retourné » accentue le fait qu'au Bénin la condition d'esclave n'est pas aussi stigmatisée que cela. Et ce d'autant moins, qu'en dehors de la communauté aguda, il y a également comme un brouillage entre victimes et acteurs de la Traite. Mais si tous les Aguda retournés au Dahomey n'ont pas été des négriers, la plupart ont participé à différents niveaux aux processus de colonisation par la France de cet ancien royaume. Et lorsque certains membres de l'élite aguda clament aujourd'hui à la face du monde leur origine esclave faisant en quelque sorte un outing à la mode anglo-saxonne, au niveau local, loin de s'affirmer comme victime de la traite atlantique, la majorité des Aguda mettent en avant leur style de vie européen et leur rôle dans l'introduction du catholicisme dans la région, y compris chez un personnage comme Urbain Karim da Silva, qui tout en étant musulman, était à la fin des années 1980, un des promoteurs de la mascarade carnavalesque bourian à Porto Novo qui se déroulait le troisième dimanche suivant la fête de l'Épiphanie et accompagnait la célébration de la fête du Seigneur de la Bonne Fin.

10 Ainsi, l'adhésion à un modèle qui célébrerait le métissage et l'hybridité, nous semble bien plus répondre à un idéal véhiculé par une élite en syntonie avec le cosmopolitisme des organismes internationaux qu'à celui du Brésilien aguda lambda. Ce que reconnaît largement A. L. Araujo dans sa conclusion en retenant le fait que la mémoire publique de l'esclavage au Bénin est étroitement liée aux phénomènes transnationaux de mémorisation de l'esclavage et de la traite atlantique. Le but étant de développer avec le retour à la démocratisation, un tourisme culturel où s'effacerait peu à peu le stigmate de la Traite en lui substituant les racines africaines retrouvées. À l'inverse, au Brésil, la mise en mémoire de l'esclavage aboutit à une politique de réparation vis-à-vis des descendants des victimes de l'esclavage.

11 Pour conclure, on regrettera que l'auteure, qui s'appuie sur une bibliographie presque exhaustive des travaux tant anglophones que francophones sur le thème de la traite atlantique et des reconfigurations identitaires dans l'espace atlantique sud - il manque cependant l'ouvrage fondamental de D. Y. Peel sur la construction des Yorubas -, laisse transparaître une approche diffusionniste des relations entre l'Afrique et le Brésil. En effet, si le XIX ${ }^{e}$ siècle est la période où les contacts entre le Brésil et le royaume d'Abomey ont été les plus intenses, du fait des voyages fréquents d'une élite afrobrésilienne en train de se constituer, répondant comme en écho à l'émergence d'une nouvelle élite africaine moderne à travers le travail salarié, il est néanmoins indubitable que la fin de l'esclavage a marqué la fin des échanges entre le Brésil et le Bénin, et ce en dépit du rêve d'une certaine élite intellectuelle brésilienne de renouer avec la terre africaine. Car comme le notait Beluce Belluccini ${ }^{1}$ à propos du Mozambique, il est légitime de s'interroger sur le fait que les Brésil et Bénin contemporains 
pourraient instrumentaliser la mémoire de l'esclavage pour développer son économie dans un esprit tout à fait capitaliste et néolibéral.

\section{NOTES}

1. <http://www.fpabramo.org.br/artigos-e-boletins/artigos/\%E2\%80\%9Cesta-terraainda-vaicumprir-seu-ideal\%E2\%80\%9D> 